\title{
Exploring Geostatistical Modeling and Visualization Techniques of Uncertainties for Categorical Spatial Data
}

\author{
Carlos A. Felgueiras, Jusssara O. Ortiz, Eduardo C. G. Camargo, Laércio M. Namikawa, Thales S. Körting \\ Instituto Nacional de Pesquisas Espaciais, Brazil \\ \{carlos.felgueiras, jussara.ortiz, eduardo.camargo, laercio.namikawa, thales.korting\}@inpe.br
}

\begin{abstract}
This article presents and analyzes the indicator geostatistical modeling and some visualization techniques of uncertainty models for categorical spatial attributes. A set of sample points of some categorical attribute is used as input information. The indicator approach requires a transformation of sample points on fields of indicator samples according to the classes of interest. Experimental and theoretical semivariograms of the indicator fields are defined representing the spatial variation of the indicator information. The indicator fields, along with their semivariograms, are used to determine the uncertainty model, the conditioned probability distribution function, of the attribute at any location inside the geographic region delimited by the samples. The probability functions are considered for producing prediction and probability maps based on the maximum class probability criterion. These maps can be visualized using different techniques. In this article, it is considered individual visualization of the predicted and probability maps and a combination of them. The predicted maps can also be visualized with or without constraints related to the uncertainty probabilities. The combined visualizations are based on three-dimensional (3D) planar projection and on the RedGreen-Blue to Intensity-Hue-Saturation (RGB-IHS) fusion transformation techniques. The methodology of this article is illustrated by a case study with real data, a sample set of soil textures observed in an experimental farm located in the region of São Carlos city in São Paulo State, Brazil. The resulting maps of the case study are presented and the advantages and the drawbacks of the visualization options are analyzed and discussed.
\end{abstract}

Categories and Subject Descriptors: D.2 [Software Engineering]: Design Tools and Techniques; I.4 [Image Processing and Computer Vision]: Enhancement; I.6 [Simulation and Modeling]: Model Development

Keywords: Indicator Geostatistics, Spatial Modeling of Categorical Attributes, Uncertainty Visualization

\section{INTRODUCTION}

Continuous and categorical spatial attributes can be computationally modelled, from a set of sample points obtained from field works or other spatial data sources, for many environmental applications in a geographical region of interest. The attribute representations are used as input for spatial modelling functions whose outputs simulate Earth related phenomena in a Geographical Information System (GIS) database, allowing to go deep in studies and analyses to support better decision makings for real world problems.

The representation of uncertainty has become a topic of interest in different visualization domains such as geographic visualization, information visualization, and scientific visualization, as well as in related domains such as Visual Analytic and decision science [Kinkeldey and Hensi 2018]. Geospatial data will always leave their user uncertain about the true nature of the world [Goodchild 2020]. Geostatistical approaches yield tools for representing the stochastic local or global uncertainty models of geographical attributes from their input sample sets. Maps of predictions, which are derived from mean, median or mode values, and related uncertainty quality maps, based on standard deviation, quantile or probability values, can be extracted from the attribute uncertainty models. So, the predictions can be accompanied with their uncertainty values that qualify the predictions and are also

Copyright(C)2021 Permission to copy without fee all or part of the material printed in JIDM is granted provided that the copies are not made or distributed for commercial advantage, and that notice is given that copying is by permission of the Sociedade Brasileira de Computação. 
spatially distributed in the region of interest. In special, using indicator geostatistical functions for interpolations and simulations, the uncertainty fields take into account the sample values and also their relative spatial locations [Deutsch and Journel 1998]. Moreover, the indicator geostatistics allow modelling uncertainties of categorical, besides the continuous, attribute information [Goovaerts 1997], [Isaaks and Srivastava 1989], [Felgueiras et al. 2015] and [Felgueiras et al. 2016].

A significant topic for the attribute representations is to visualize their uncertainty fields in a way that facilitates the analysis of the spatial distribution in terms of quality of the attribute modelling [Foody and Atkinson 2002]. Many scientific articles have been addressed the subject of visualization methods to represent spatial data uncertainties [Pebesma et al. 2007], [Tan and Chen 2008], [Sun and Wong 2010], [Senaratne et al. 2012], [Deitrick and Wentz 2015], [Ślusarski and Jurkiewicz 2020], [PérezDíaz et al. 2020]. Decision makers usually deal with uncertainties based on how current conditions or policies affect the future results in their decision. But, a comprehensive geovisualization of uncertainty in spatial data can be enhanced by providing a visual representation of their attribute uncertainty, which in turn can assist users to more easily recognize an underlying pattern of data [Koo et al. 2018].

Typically, an uncertainty map is visualized separately from the data map using a gray scale look up table (LUT) where the minimum and maximum values are assigned to the black and white colors respectively, or vice-versa. The intermediary colors are defined as midway gray levels proportional to the attribute values. It is also common to show the uncertainty maps using different color tables, as the rainbow colors for example.

[Koo et al. 2015] present a framework for combining visual variables to represent simultaneously an attribute and its uncertainty. The authors use three categories of uncertainty visualizations: coloring, overlap symbols and integrate symbols. [Koo et al. 2018] has implemented the framework for attribute uncertainty visualization as an extension of the software known as ArcGis, mainly for choropleth maps and proportional symbol maps for thematic mapping of attributes. Another interesting approach is to use fusion techniques to visualize the attribute data merged with their quality for displaying both information integrated in a single map. [Hengl 2003] describes two methods for visualization of uncertainty associated with predictions of continuous and categorical variables. Both methods are based on the Intensity-Hue-Saturation (IHS) color model with uncertainty coded with whiteness. Also, in a GIS environment, it is common to obtain 3D planar projections of attribute representations and to use the uncertainty as the texture of the rendered images.

In this context, the objective of this article is to explore the modelling and visualization the uncertainties of categorical spatial attributes. The uncertainty modelling is performed by procedures of indicator geostatistics applied to a sample set of points of a spatial categorical attribute. It is considered the following visualization techniques: individual visualization of the uncertainty maps, with and without probability constraints; visualization of the predictions combined with their uncertainties using 3D planar projections; and visualization resulting from fusion technique based on Red-Green-Blue to Intensity-Hue-Saturation (RGB-IHS) color transformation. The methodology is illustrated with a case study that utilize a punctual sample set of soil texture observed in an experimental farm located in the region of São Carlos city in São Paulo State, Brazil. Four classes of soil texture, namely Sandy, Medium Clayey, Clayey and Too Clayey, are considered in order to obtain the predictions along with their uncertainties. The paper presents and analyses the resulting maps of this case study and the advantages and the drawbacks of the visualization options are discussed. This article is an extended version of [Felgueiras et al. 2017] presented in XVIII Brazilian Symposium on GeoInformatics.

The organization of this article starts with this introduction section. Section 2 presents summaries of the main concepts linked to the main issues of this research. Section 3 addresses the methodology of this research while section 4 describes the case study used to illustrate the modelling and visualizations resulting from application of the proposed methodology. Section 5 shows results and analyses related to the adopted attribute uncertainty modelling and map visualizations. In the section 6 , conclusions are reported along with suggestions for future researches. 


\section{CONCEPTS}

\subsection{Indicator Geostatistics}

Geostatistical procedures can be used to generate statistical uncertainty models of spatial attributes and, from them, to derive attribute realizations, predictions (such as mean, median and mode values) and uncertainty metrics based on probabilities and confidence intervals of standard deviations and quantiles.

The geostatistical indicator approaches allow modeling the joint conditional distribution functions of continuous or categorical random variables, at any unknown spatial location $\mathbf{u}$, considering an available sample points set. The Simulation process consists of drawing realizations from the joint conditional distribution functions.

For a categorical variable, its conditional probability distribution function (cpdf) is built from estimations on indicator fields obtained by indicator transformations applied to the original sample set, of nsamples, $z(\mathbf{u} j), j=1, \ldots, n s a m p l e s$, considering any number of nclasses. Instead of the variable $Z\left(\mathbf{u}_{j}\right)$, consider its binary indicator transformation $I\left(\mathbf{u}_{j} ; k\right)$ as defined by the relation of Equation 1.

$$
I\left(\mathbf{u}_{j} ; k\right)= \begin{cases}1, & \text { if } Z\left(\mathbf{u}_{j}\right)=k \\ 0, & \text { otherwise }\end{cases}
$$

This transformation is equivalent to associate probability $1(100 \%)$ for $Z\left(\mathbf{u}_{j}\right)$ values which are equal to class $k$ and 0 otherwise. The result of transformation expressed in Equation 1 generates $k$ indicator fields, with 0 and 1 values, $i\left(\mathbf{u}_{j}\right), j=1, \ldots$, nsamples of the indicator variable $I\left(\mathbf{u}_{j} ; k\right)$. Next, experimental indicator semivariograms, $\widehat{\gamma}$, are defined, from the Equation 2, for each one of the $k$ indicator fields to represent their spatial variations:

$$
\widehat{\gamma}_{(\mathbf{h} k)}=\frac{1}{2 N(\mathbf{h})} \sum_{j=1}^{N(\mathbf{h})}\left[i\left(\mathbf{u}_{j} ; k\right)-i\left(\mathbf{u}_{j}+\mathbf{h} ; k\right)\right]
$$

where $i\left(\mathbf{u}_{j} ; k\right)$ and $i\left(\mathbf{u}_{j}+\mathbf{h} ; k\right)$ are the $j$-th values of the indicator variable $I$, separated by the distance vector $\mathbf{h}$, and $N(\mathbf{h})$ is the number of the pairs of points that are separated by $\mathbf{h}$.

The $\mathrm{k}$ indicator fields and their respective theoretical semivariograms are used by the kriging procedure for assessment of class probabilities at any spatial location inside the region of interest. Moreover, the sequential indicator simulation procedure uses kriging, applied on the indicator sample and pre-realization values, in order to infer the $c p d f s$ and the class realizations of the categorical variable ([Goovaerts 1997] and [Goovaerts 2001]). Maps of predictions with $c^{*}\left(\mathbf{u}_{\alpha}\right)$ and uncertainties $U n c^{*}\left(\mathbf{u}_{\alpha}\right)$ values, based on local maximum probabilities $P_{k}(\mathbf{u})$ of any $c p d f$, can be evaluated from the set of realization fields as presented by the Equations 3 and 4 .

$$
\begin{gathered}
c^{*}\left(\mathbf{u}_{\alpha}\right)=c_{l}\left(\mathbf{u}_{\alpha}\right) \quad \text { where } P\left(c_{l}\left(\mathbf{u}_{\alpha}\right)\right)>P\left(c_{k}\left(\mathbf{u}_{\alpha}\right)\right) \quad \forall l, k=1 \ldots \text { nclasses } \\
\operatorname{Unc}^{*}\left(\mathbf{u}_{\alpha}\right)=1-\operatorname{Max}\left(P\left(c_{k}\left(\mathbf{u}_{\alpha}\right)\right)\right) \quad k=1 \ldots \text { nclasses }
\end{gathered}
$$

In Equations 3 and $4, \mathbf{u}_{\alpha}, \alpha=1, . .$, gridsize(nrowsxncolumns), are spatial locations regularly distributed in the geographical space, determining regular grid representation structures.

The results of Equation 3 can also be constrained by a threshold of uncertainty probability TP, as show in Equation 5.

$$
c^{* *}\left(\mathbf{u}_{\alpha}\right)=c^{*}\left(\mathbf{u}_{\alpha}\right) \text { where } \operatorname{Unc}^{*}\left(\mathbf{u}_{\alpha}\right)<\mathrm{TP}
$$

Moreover, the maps produced from equations 3 and 5 can be limited to a number of classes of interest smaller than the total number of input classes. 


\subsection{D Planar Projections}

The 3D planar projections allow the visualization of 3D information in a 2D surface using geometric transformations. Parallel projections position the viewpoint at the infinite while when the viewpoint is elsewhere the projections are known as perspective projections. The 3D planar projections are generally based on applying geometric transformations based on Translations, Scaling and Rotations. Many books of basic computer graphics, such as [Newman and Sproul 1978] and [Foley et al. 1995], present details of the mathematics evolved on this subject. Rendering planar projections of 3D information considers hidden lines or surfaces and inclusion of additional 2D texture information to accomplish more realistic 2D images. As an example, Figure 1 depicts the 3D Planar Projection of a Shuttle Radar Topography Mission (SRTM) elevation grid using wired and textured parallel projections.

\subsection{RGB - IHS Transformations}

The human eye perceives color information through three types of cones with sensitivity to the Red $(\mathrm{R})$, Green $(\mathrm{G})$ and Blue (B) wavelengths of visible electromagnetic energy. This physical schema is the base of the RGB color system, where individual intensities of Red, Green and Blue combine to define a color. In terms of human perception, it is more natural to evaluate the values Intensity (I), the Hue $(\mathrm{H})$ and the Saturation $(\mathrm{S})$ of a color. Intensity corresponds to the total energy measure involved in all wavelengths and provides the brightness sensation. The Hue is the average wavelength of the light and determines the object color. Saturation expresses the purity of the color with low saturation values producing pale tones and high saturation values presenting pure colors. The IHS color system is also known by other names, depending on how the Intensity component is named: HSV system has Value (V) for Intensity; and HLS has Lightness (L) for Intensity (there is a slight change in this system but the overall idea is the same). There are different ways to perform the RGB-IHS transformation, and vice-versa, mostly presented in books of Computer Graphics, such as [Foley et al. 1995] for example.

The RGB-IHS and IHS-RGB color transformations are widely used in remote sensing applications to fuse images of different resolutions and/or sensors. Three bands from a multispectral image are selected and associated to a corresponding RGB component and then transformed into IHS model. Next, in the IHS-RGB reverse transformation the process replaces one of the IHS components. Usually, the intensity component is replaced by one panchromatic band with higher resolution when a pansharpening fusion is required. In this case, the resulting RGB components present an enhanced image with higher spatial resolution with the colors of the multispectral image. In this research, we explore this fusion procedure using the spatial attribute predicted image, as the input multispectral image, and it respective uncertainty as auxiliary information that will replace the Intensity and Saturation components.
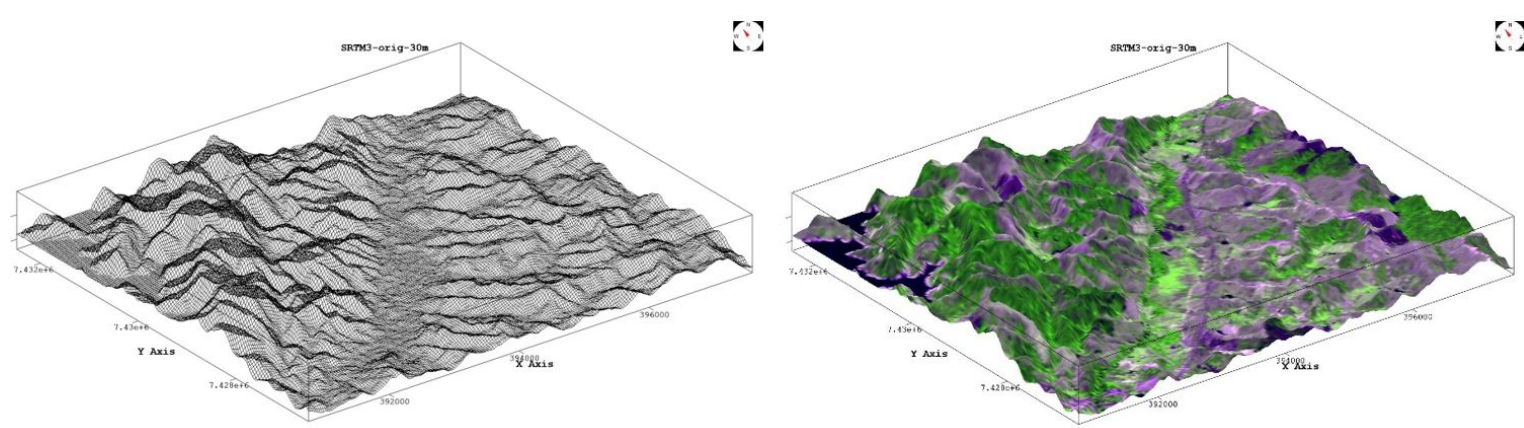

Fig. 1. Planar projection of a SRTM grid data: wired (left) and rendered with texture of a remote sensing image (right) 


\section{METHODOLOGY}

Considering specifically spatial categorical attributes, the methodology of this research, for their uncertainty modelling and visualizations, comprises the following sequence:

-A sampling set of points of the categorical attribute, given as the input data, is initially transformed in indicator sample sets according to the number of classes considered.

-Experimental and theoretical semivariograms are obtained for the indicator sample fields to represent their respective spatial variability.

- The indicator fields and their theoretical semivariograms are used to run Sequential Indicator Simulation (SIS) functions of the Geostatistical Software Library (GSLib) [Deutsch and Journel 1998], in order to obtain realization values and from them prediction and uncertainty maps of the attribute in the spatial region of interest.

- Prediction and uncertainty maps are visualized individually using different lookup tables and constraints.

- Prediction and uncertainty maps are combined in 3D planar projection visualizations.

- RGB-IHS transformation is applied in the Red, Green and Blue components of the predicted categorical map.

- IHS-RGB reverse transformation is applied by replacing the Intensity or the Saturation component by the Uncertainty map.

- RGB layers from the reverse transformation are combined in order to display and compare the results of the fusion processes.

\section{A CASE STUDY}

In order to illustrate the proposed methodology, a set of points of soil texture data sampled in the region of an experimental farm known as Canchim is used. This region of interest is located at the city of São Carlos, São Paulo, Brazil, and covers an area of 2660 ha between the north-south coordinates from $s 21^{\circ} 54^{\prime} 32$ " to $s 21^{\circ} 59^{\prime} 39^{\prime \prime}$ and the east-west coordinates from $w 47^{\circ} 48^{\prime} 11^{\prime \prime}$ to $w 47^{\circ} 51^{\prime} 59^{\prime \prime}$.

The input data set consists of 86 samples of soil texture information each classified as one of the following four classes: Sandy, Medium Clayey, Clayey or Too Clayey. Figure 2 illustrates the borders of the Canchim farm and the spatial locations, black * marks inside the region, of the classified soil texture samples. The classified map, used as background of this figure, is obtained by nearest neighbor estimations showing regions of influence of each class. The SPRING GIS [Camara et al. 1996] is used to store, analyze and visualize all the geoinformation of this case study.

\section{RESULTS AND DISCUSSIONS}

\subsection{Soil texture estimated by Indicator Geostatistics}

The spatial dependence analyses are based on the indicator sample fields of the soil texture classes generated by the indicator transformation as defined in Equation 1.

The spatial dependencies are represented by the indicator semivariograms generated from the indicator sample set defined by each texture class. The experimental indicator semivariograms are assessed and fitted by theoretical ones in the SPRING GIS and the PyESSDA [Felgueiras et al. 2019] computational environment. The indicator semivariogram parameters, along with the global probabilities of each texture class, are reported in Table I. All semivariograms are fitted with exponential functions. The global probabilities are assessed by the ratio between the number of samples of each class and the total number. These parameters and the sample set are used as input for the SIS function. 


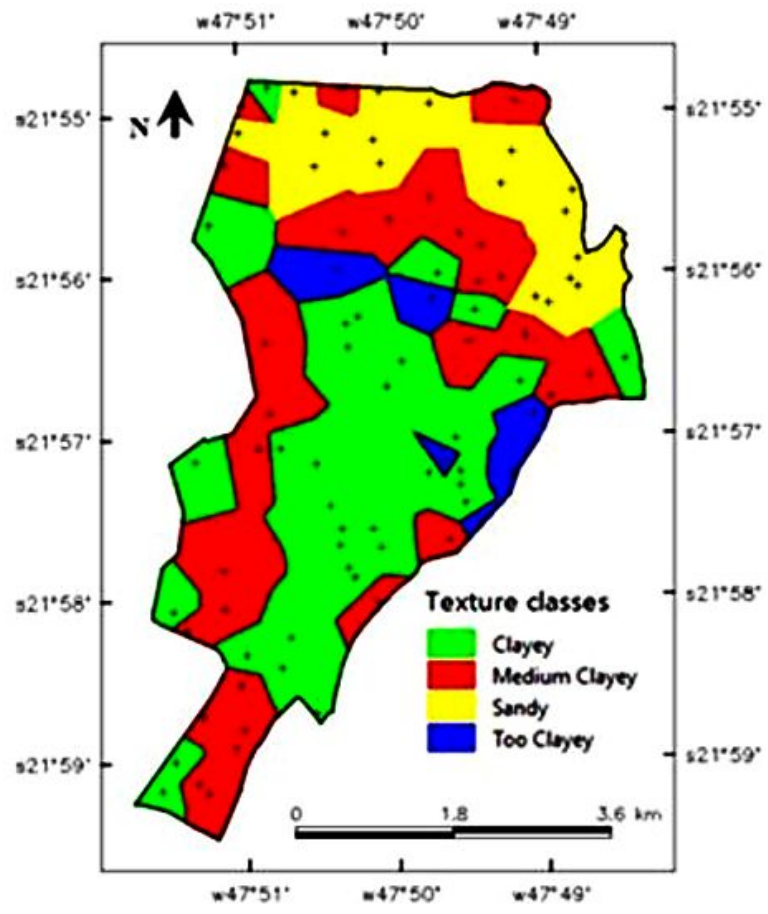

Fig. 2. Distribution of the soil texture sample points within the Canchim region

Figure 3(a) shows the map of predicted soil texture classes while Figure 3(b) shows the map of their uncertainties. These maps are obtained from the resulting realizations of the GSLib SIS function known as sisim. Those estimations are assessed from the cpdfs' higher probability criterion, as in Equations 3 and 4, for each spatial location considered.

A visual qualitative comparison between the map of predictions in Figure 3(a) and the map of nearest neighbors' interpolation in Figure 2 shows that both maps agree with the local information presented in the texture sample set. The differences appear in the smoother class transitions presented in the map predicted from the geostatistical simulated values.

As expected for environmental attributes, the uncertainties depicted in Figures 3(b) and 3(c) are higher at the borders and at the transition areas of the soil texture class regions. Consequently, the probability uncertainty values are lower in the middle of each map class. It is possible also to observe that the minimum uncertainty values appear at the sample locations since the geostatistical procedures are exact, i.e., the estimation is equal to the sample value at any sample location. Figure 3(c) depicts the map of uncertainties using a rainbow look up table color. The map in Figure 3(b), compared with the one in Figure 3(c), seems to be better to emphasize borders and transitions between classes where the uncertainties are higher, among classes of the predicted map. Other lookup tables can be used in order to highlight specific details.

Table I. Parameters of Indicator Semivariograms related to the Soil Texture Classes

\begin{tabular}{|c|c|c|c|c|}
\hline Texture Class & Nugget Effect & Contribution & Range & Global Probability \\
\hline Sandy & 0.0 & 0.14 & 1915 & 0.20 \\
\hline Medium Clay & 0.0 & 0.22 & 902 & 0.35 \\
\hline Clayey & 0.01 & 0.20 & 1059 & 0.38 \\
\hline Too Clayey & 0.03 & 0.05 & 695 & 0.07 \\
\hline
\end{tabular}



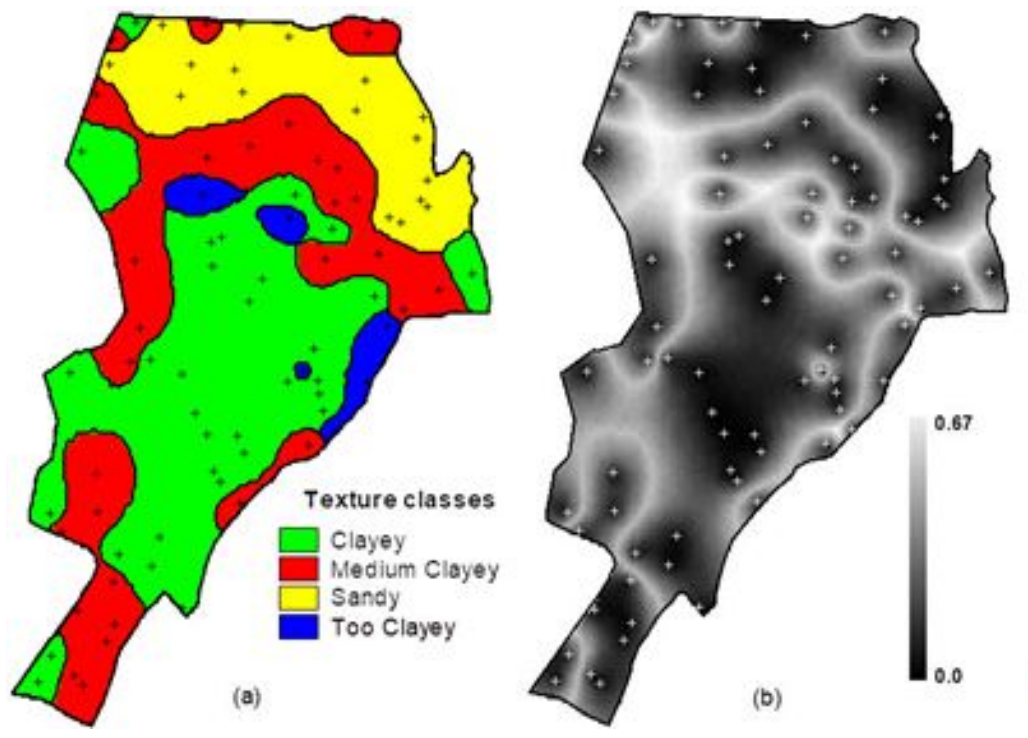

(b)

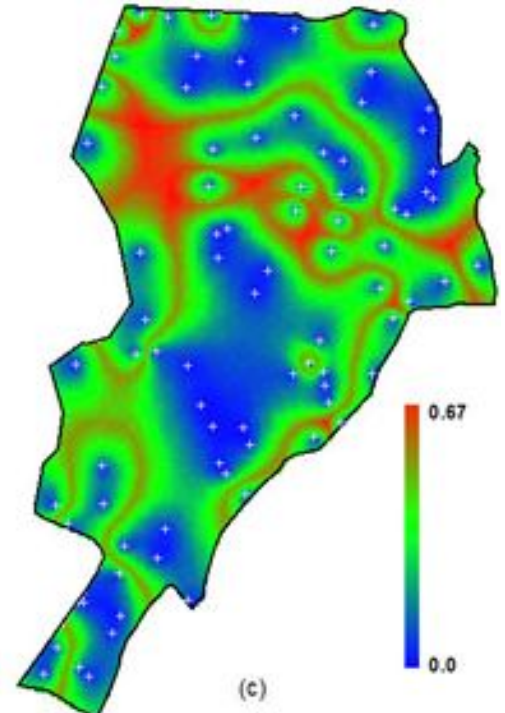

(c)

Fig. 3. Map of (a) predictions, (b) uncertainties in a gray scale LUT and (c) uncertainties in a rainbow LUT

Also, the information of predictions can be constrained to the probabilities and to the number of classes. Figure 4 and 5 show the maps of predictions constrained to probability uncertainty thresholds of .5 and .25 . This means that the areas of the original classes maintained on the maps in Figures 4a and $4 \mathrm{~b}$ represent regions with at least 50 and 75 percent of certainty probability, respectively. The maps in Figure 4 use all the input classes while in Figure 5 only the classes medium clayey and clayey are taken into account. In short, it is possible to visualize the maps of prediction with or without constraints, depending on the decision making processes requested for the considered application.
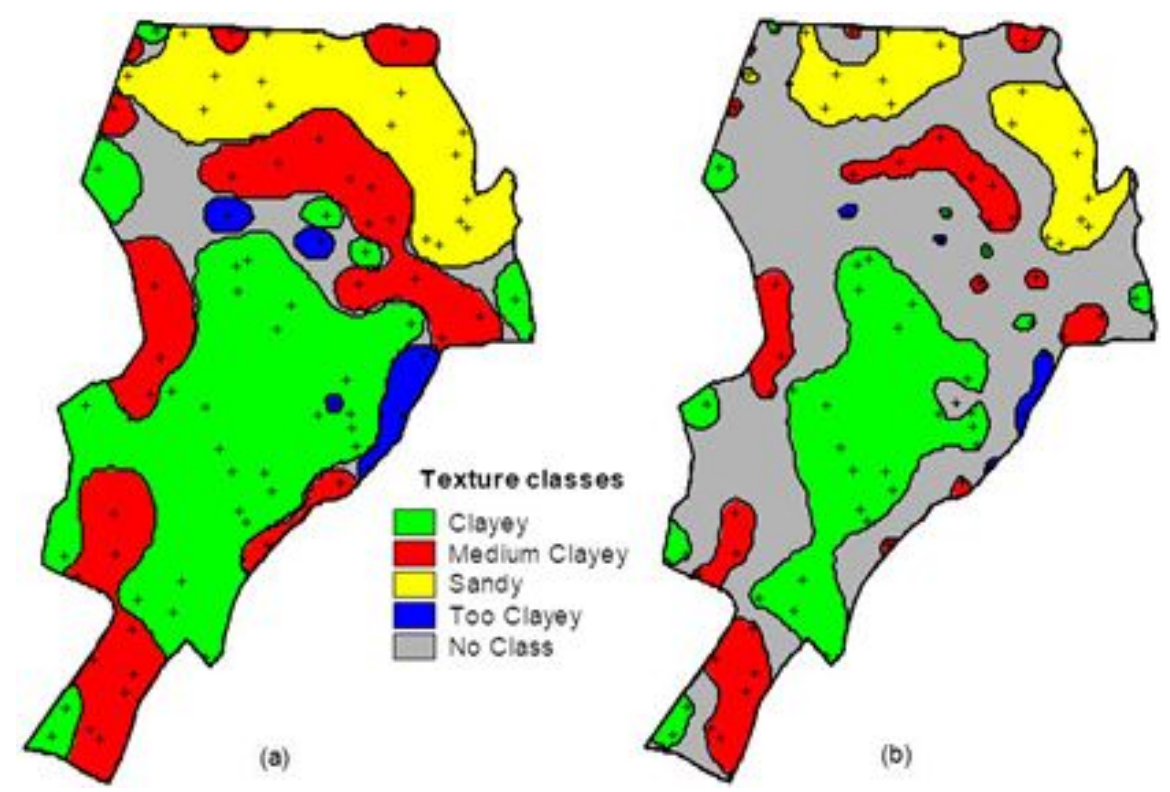

Fig. 4. Prediction maps of all texture classes constrained by different uncertainty threshold values: (a) .5 and (b) .25 (adapted from [Felgueiras et. al. 2016]) 

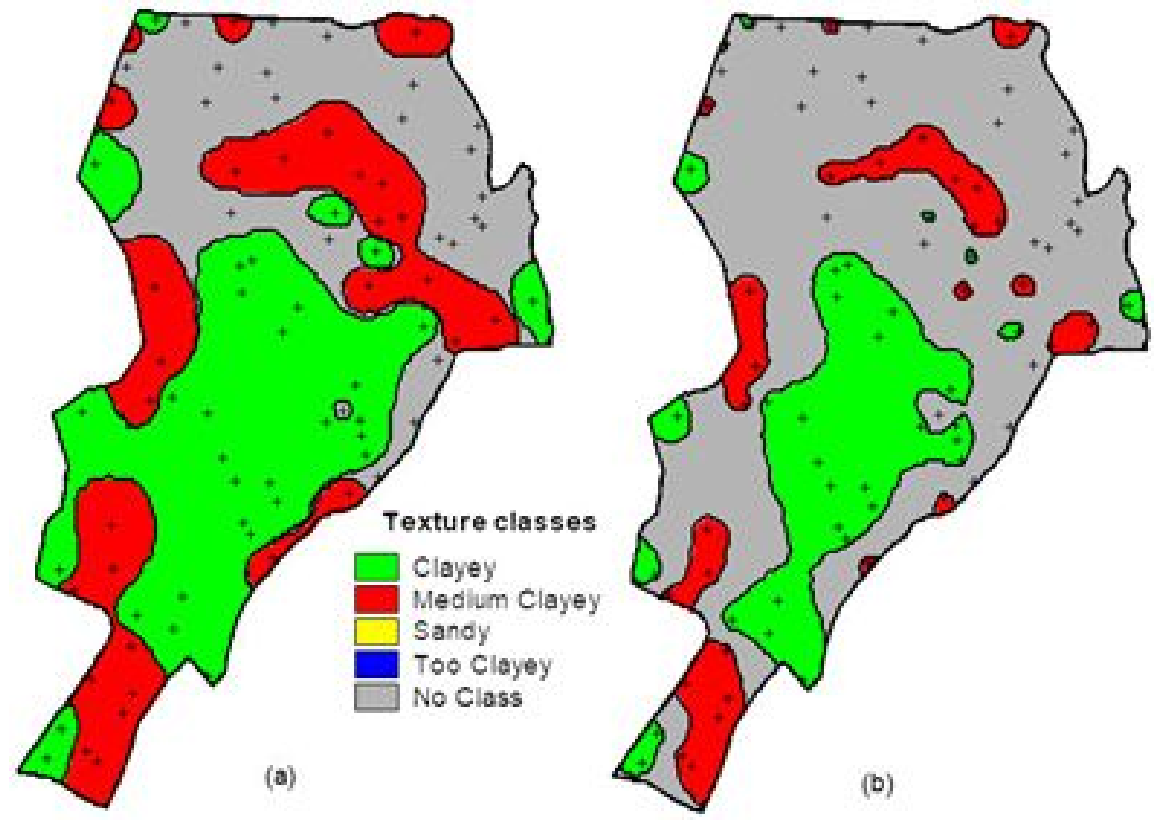

Fig. 5. Prediction maps of medium clayey and clayey texture classes constrained by different uncertainty threshold values: (a) .5 and (b) .25 (adapted from [Felgueiras et. al. 2016])

\subsection{Uncertainty visualization by $3 \mathrm{D}$ planar projection}

Figure 6(a) displays the uncertainty information in a 3D Planar Projection using the gray level map of Figure 2(b) as the texture of the final rendered figure while in Figure 6(b) the texture is gathered from the predicted map in Figure 2(a). Figures 6(a) and 6(b) can also be rendered using different azimuth and zenith angles and are considered qualitative applications.

These drawings are useful in order to have a visual perception of the vertical variation of the uncertainty information at different angles together with other soil texture information. Other textures can be used, e.g., the one of Figure 2(c), 4(a), 4(b), 5(a) or 5(b).
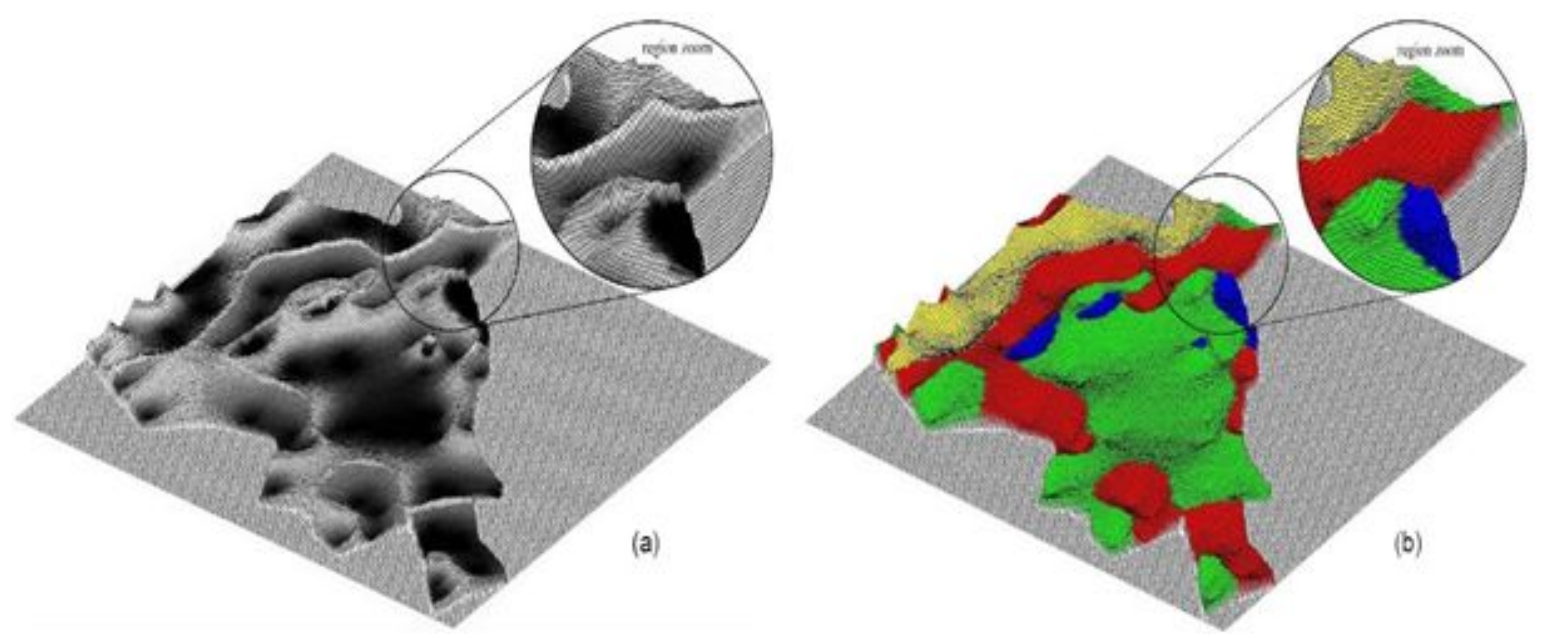

Fig. 6. Planar projections of the uncertainty map displayed in (a) gray levels and (b) predicted classes as texture of the rendered map 

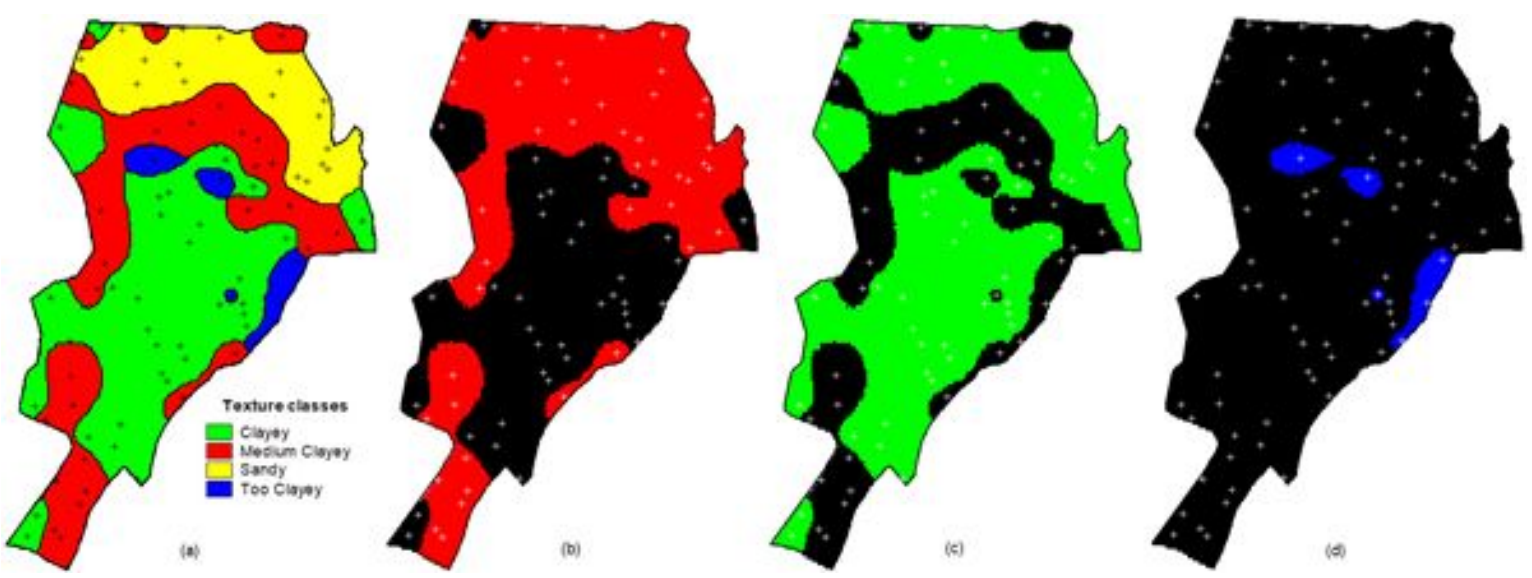

Fig. 7. Map of (a) Predictions, (b) Red component, (c) Green component and (d) Blue component

\subsection{Uncertainty Visualization by RGB-IHS fusion}

All images that has been considered in this application are color coded with 8 bits, so the minimum and the maximum values for colors are 0 and 254 . The 255 value is used as the background color. The texture classes have the following R, G, B color composition: Sandy 254, 254, 0 (Yellow); Medium Clayey 254, 0, 0 (Red); Clayey 0, 254, 0 (Green) and; Too Clayey 0, 0, 254 (Blue). Taking into account its class colors, the predicted texture map can be decomposed in three new maps corresponding to the Red, Green and Blue components. These maps are presented in Figure 7(a) to 7(d).

Applying the RGB-IHS transformation to the components of the RGB texture map results in a Saturation component equal to 254, the maximum value, for the entire region. The Intensity component is assigned to 127 , a medium value. The Hue component varies according the colors presented in the predicted map. Low Hue values (Black) represents the yellow color, low medium values Green, high medium values Blue and high values Red. The prediction map and its IHS components are shown in Figure 8(a) to 8(d).

Figure 9 depicts the results of the IHS-RGB fusion transformation replacing Intensity and Saturation by the Uncertainty map of Figure 2(b). In Figure 9(b), the original colors of the predicted map tend to darker colors where the uncertainty is very small. This occurs because any color with low intensity appears as black. To avoid the dark colors, one could remap the uncertainty interval values to larger
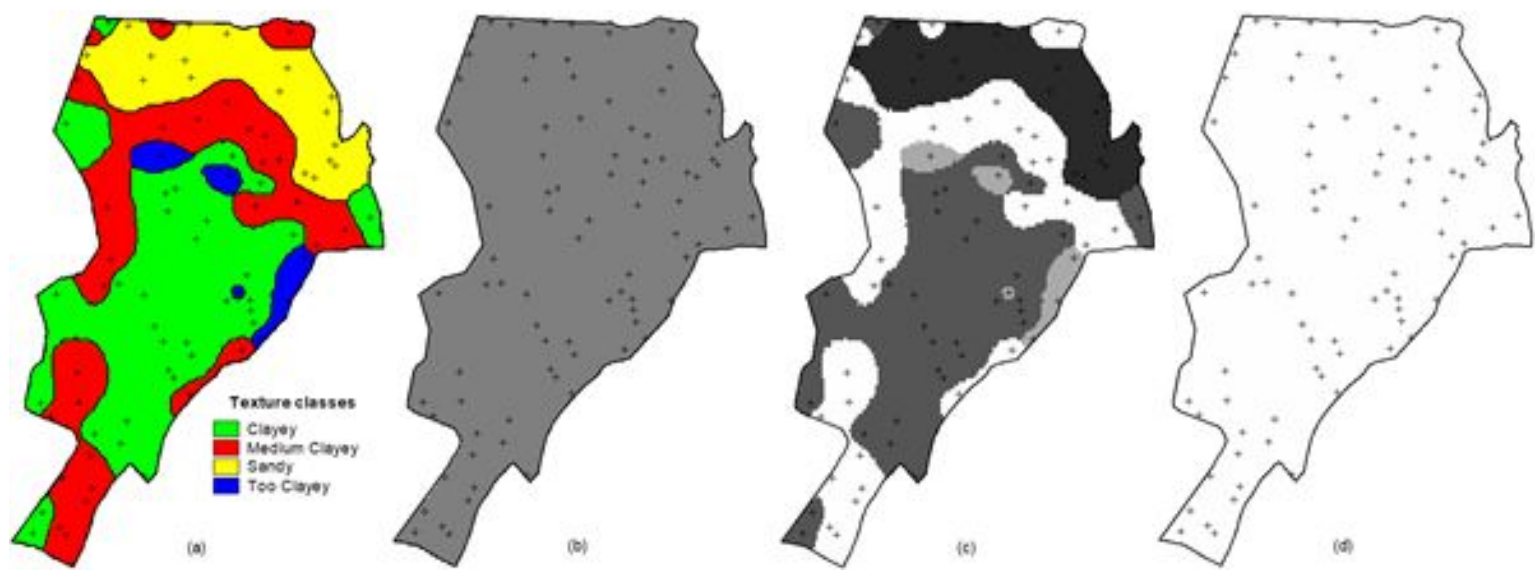

Fig. 8. Map of (a) Predictions, (b) Intensity component, (c) Hue component and (d) Saturation component 

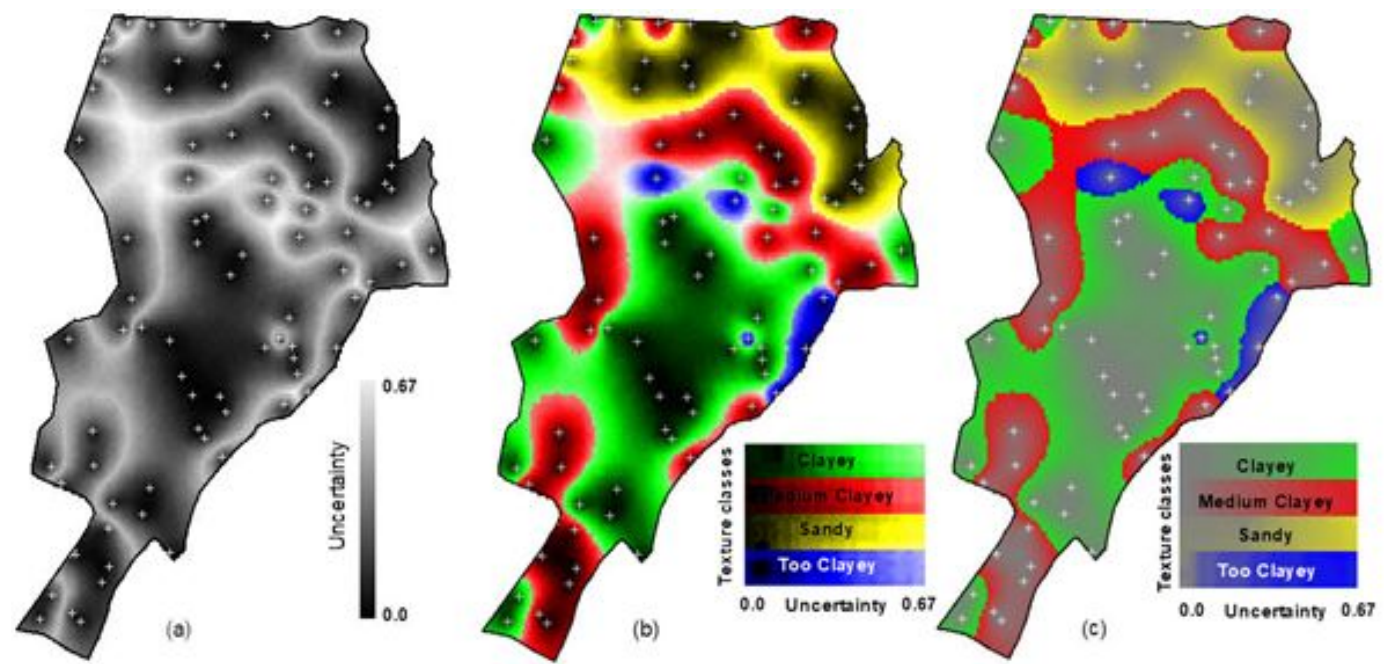

Fig. 9. IHS-RGB fusion transformation showing: (a) Uncertainty map and; (b) and (c) Intensity and Saturation replaced by the Uncertainty map respectively

values. In any case the map in Figure 9(b) shows the predominant colors of each class going from low intensities, where uncertainties are low, to high intensities, where uncertainty values are larger.

The map in Figure 9(c) has similar behavior as the one in Figure 9(b) where Saturation is considered instead of Intensity. In this case, low saturated colors appear at locations with small uncertainties and the colors appear whitened or paled. Also, here it is possible to use a remap interval of uncertainty to avoid too paled effects. Using the Saturation component, the original predicted colors seem to be preserved better than when the Intensity is considered. Moreover, the maps in Figure 9(b) and 9(c) can be rendered using the inverted uncertainty information as shown in Figure 10(a).

This can be done after applying an inverted linear function, mapping 0 to 254 and vice-versa, in the uncertainty map of Figure 9(a) before the fusions. The results of using inverted uncertainties are shown in Figure 10(b) and 10(c) where black and paled regions appear at the class transitions
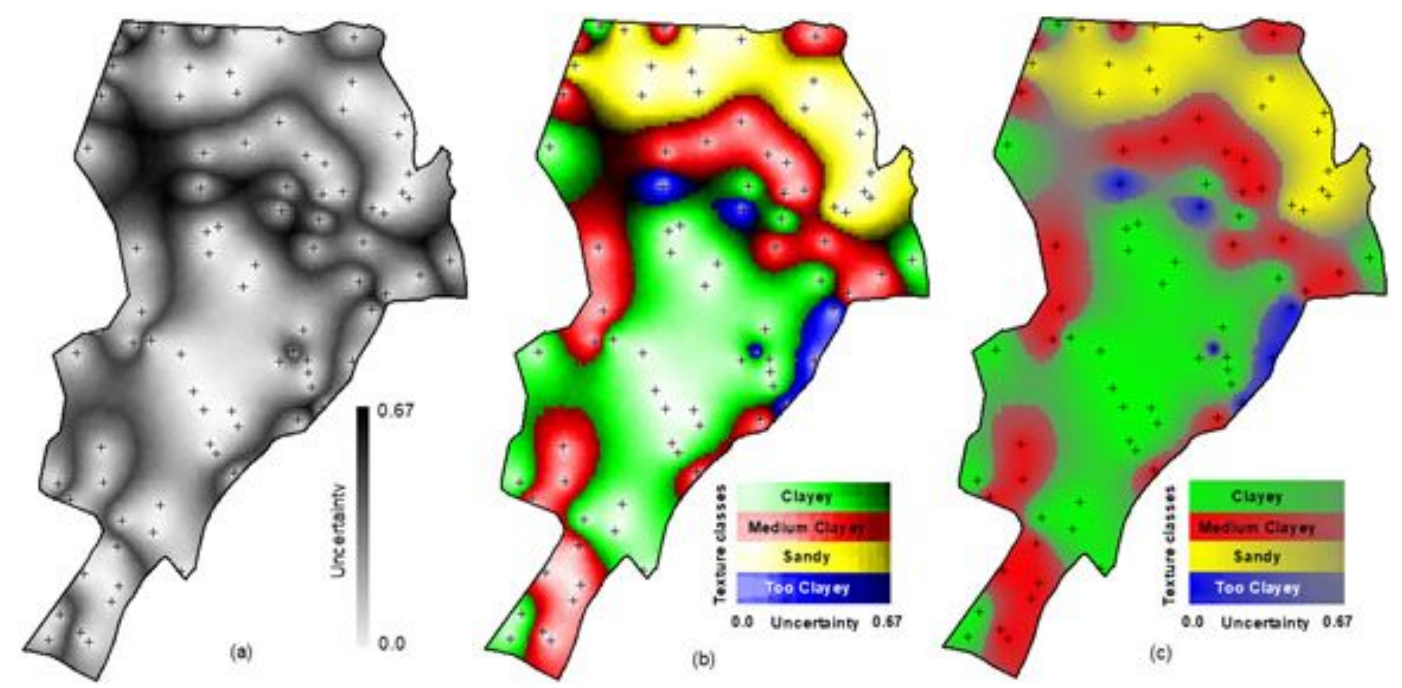

Fig. 10. IHS-RGB fusion transformation showing: (a) Inverted Uncertainty map and; (b) and (c) Intensity and Saturation replaced by the Inverted Uncertainty map respectively 
where the uncertainty values are larger. These images keep their original color classes, or saturate in white, where the uncertainty values are smaller. Although the effectiveness of the above visualization methods has not yet been evaluated by a substantial number of users, it suggests that the maps in Figures $8(\mathrm{~b}), 8(\mathrm{c}), 9(\mathrm{~b})$ and $9(\mathrm{c})$ allow one to have an integrated perception of both information, the predicted colors or classes and the uncertainties, mixed in the same map. Furthermore, these maps can be used as background of cartographic charts in order to enhance their final presentation, for example. Also, it is possible to use the fusioned maps as texture information for the $3 \mathrm{D}$ planar projection as presented in Figure 5.

\section{CONCLUSIONS}

This article presents and analyzes the indicator geostatistical modeling and some visualization techniques of uncertainty models for categorical spatial attributes. A set of sample points of some categorical attribute is used as input information. The indicator approach requires a transformation of sample points on fields of indicator samples according to the classes of interest. Experimental and theoretical semivariograms of the indicator fields are defined representing the spatial variation of the indicator information. The indicator fields, along with their semivariograms, are used to determine the uncertainty model, the conditioned probability distribution function, of the attribute at any location inside the geographic region delimited by the samples. The probability functions are considered for producing prediction and probability maps based on the maximum class probability criterion. These maps can be visualized using different techniques. In this paper, it is considered individual visualization of the predicted and probability maps and a combination of them. The predicted maps can also be visualized with or without constraints related to the uncertainty probabilities. The combined visualizations are based on three-dimensional (3D) planar projection and on the Red-Green-Blue to Intensity-Hue-Saturation (RGB-IHS) fusion transformation techniques. The methodology of this article is illustrated by a case study with real data, a sample set of soil textures observed in an experimental farm located in the region of São Carlos city in São Paulo State, Brazil. The resulting maps of the case study are presented and the advantages and the drawbacks of the visualization options are analyzed and discussed. For planning purposes, all the maps that has been shown in this article can be considered for decision making activities when an application manager aims to save financial and/or environmental resources. In the future, we intend to explore similar methodology for spatial modelling and visualization of spatial continuous attributes and other fusion techniques.

\section{Acknowledgments}

The authors would like to thank the support of the São Paulo Research Foundation (FAPESP, process number 2015/24676-9) and also thank the Brazilian National Council for Scientific and Technological Development (CNPq, grant number 303360/2019-4).

\section{REFERENCES}

Camara, G., Souza, R. C. M., Freitas, U. M., and Garrido, J. SPRING: Integrating Remote Sensing and GIS by Object-Oriented Data Modelling. Computer \& Graphics 20 (3): 395-403, 1996.

Deitrick, S. and Wentz, E. A. Developing Implicit Uncertainty Visualization Methods Motivated by Theories in Decision Science. Association of American Geographers 105 (3): 531-551, 2015.

Deutsch, C. V. and Journel, A. G. GSLIB: Geostatistical Software Library and User's Guide. Oxford University Press, Oxford, New York, 1998.

Felgueiras, C. A., Monteiro, A. M. V., Ortiz, J. O., and Camargo, E. C. G. Improving Accuracy of Categorical Attribute Modelling with Indicator Simulation and Soft Information. In Proceedings of International Conference on GeoComputation. Springer International Publishing, Richardson, Texas, USA, pp. 25-31, 2015.

Felgueiras, C. A., Ortiz, J. O., and Camargo, E. C. G. Spatial Predictions of Categorical Attributes Constrained to Uncertainty Assessments. In Libro de Actas del Simposio Internacional en Percepción Remota y Sistemas de Información Geográfica. EdUnLu - Editorial Universidad Nacional de Luján, Puerto Iguazú, Misiones, Argentina, pp. 1731-1740, 2016. 
Felgueiras, C. A., Ortiz, J. O., and Camargo, E. C. G. An User-friendly Python Application for Exploratory and Structural Spatial Dependence Analysis for Sample Points of Spatial Attributes. In GeoInfo 2019 - XVIII Brazilian Symposium on GeoInformatics. Instituto Nacional de Pesquisas Espaciais (INPE), São José dos Campos, São Paulo, Brazil, pp. 25-35, 2019.

Felgueiras, C. A., Ortiz, J. O., Camargo, E. C. G., Namikawa, L. M., and Körting, T. S. Modeling and Visualization of Uncertainties of Categorical Spatial Data using Geostatistics, 3D Planar Projections and Color Fusion Techniques. In GeoInfo 2017 - XVIII Brazilian Symposium on GeoInformatics. Instituto Nacional de Pesquisas Espaciais (INPE), Salvador, Bahia, Brazil, pp. 152-163, 2017.

Foley, J. D., van Dam, A., Feiner, S. K., and Hughes, J. F. Computer Graphics: Principles and Practice. Addison Wesley Longman, MA, United States, 1995.

Foody, G. M. And Atkinson, P. M. Uncertainty in Remote Sensing and GIS. John Wiley \& Sons, Ltd, West Sussex, England, 2002.

Goodchild, M. How well do we really know the world? uncertainty in giscience. Journal of Spatial Information Science vol. 20, pp. 97-102, 2020. Publisher Copyright: (C) by the author(s).

Goovaerts, P. Geostatistics for Natural Resources Evaluation. Oxford University Press, New York, USA, 1997.

Goovaerts, P. Geostatistical Modelling of Uncertainty in Soil Science. Geoderma 103 (1-2): 3-26, 2001.

Hengl, T. Visualization of Uncertainty using the HSI Color Model: Computations with Colors. In Proceedings of International Conference on GeoComputation. Taylor and Francis, Southhampton, United kingdom, 2003.

Isaaks, E. H. And SRivastava, R. M. An Introduction to Applied Geostatistics. Addison-Wesley, Massachussets, USA, 1989.

Kinkeldey, C. And Hensi, S. Representing uncertainty. the geographic information science \& technology body of knowledge (2nd quarter 2018 edition). https://doi.org/10.22224/gistbok/2018.2.3, 2018.

Koo, H., Chun, Y., And Griffith, D. A. Geovisualization of Attribute Uncertainty. In Proceedings of International Conference on GeoComputation. Springer International Publishing, Richardson, Texas, USA, pp. 230-236, 2015.

Koo, H., Chun, Y., And Griffith, D. A. Geovisualizing Attribute Uncertainty of Interval and Ratio Variables: a Framework and an Implementation for Vector Data. Journal of Visual Language Computation vol. 44, pp. 89-96, 2018.

Newman, W. M. And Sproul, R. F. Principles of Interactive Computer Graphics. McGraw-Hill College, New York, USA, 1978.

Pebesma, E. J., de Jonga, K., And Briggs, D. Interactive Visualization of Uncertain Spatial and Spatio-Temporal Data Under Different Scenarios: an Air Quality Example. International Journal of Geographical Information Science 21 (5): 515-527, 2007.

Pérez-Díaz, L., Alcalde, J., And Bond, C. E. Introduction: Handling uncertainty in the geosciences: identification, mitigation and communication. Solid Earth 11 (3): 889-897, 2020.

Senaratne, H., Gerharz, L., Pebesma, E., and Schwering, A. Usability of Spatio-Temporal Uncertainty Visualization Methods. In J. Gensel, D. Josselin, and D. Vandenbroucke (Eds.), Bridging the Geographic Information Sciences. Springer, Heidelberg, Berlin, pp. 3-23, 2012.

Sun, M. And Wong, D. W. S. Incorporating Data Quality Information in Mapping American Community Survey Data. Cartography and Geographic Information Science 37 (4): 285-299, 2010.

TAn, M. Z. And Chen, J. Visualization of Uncertainty Associated with Spatial Prediction of Continuous Variables using HSI Color Model: a Case Study of Prediction of $\mathrm{pH}$ for Topsoil in Peri-Urban Beijing, China. Journal of Forestry Research 19 (4): 319-322, 2008.

ŚluUsarski, M. AND JURKIEWicz, M. Visualisation of spatial data uncertainty. a case study of a database of topographic objects. ISPRS International Journal of Geo-Information 9 (1): 1-15, 2020. 